\title{
Enhancement patterns in the venous phase of contrast-enhanced ultrasounds: diagnostic value for patients with solid pancreatic lesions
}

\author{
Xueqi Chen, Fengzhi Hao, Yang Gui, Jing Zhang, Li Tan, Mengsu Xiao, Qing Zhang, Hua Meng, \\ Jianchu Li, Yuxin Jiang, Ke Lv \\ Department of Ultrasound, Peking Union Medical College Hospital, Peking Union Medical College \& Chinese Academy of Medical Sciences, \\ Beijing, China
}

Contributions: (I) Conception and design: K Lv, Y Jiang, X Chen; (II) Administrative support: H Meng, J Li; (III) Provision of study materials or patients: X Chen, Y Gui, J Zhang, L Tan, M Xiao, Q Zhang, K Lv; (IV) Collection and assembly of data: X Chen, F Hao; (V) Data analysis and interpretation: X Chen, F Hao; (VI) Manuscript writing: All authors; (VII) Final approval of manuscript: All authors.

Correspondence to: Ke Lv. Department of Ultrasound, Peking Union Medical College Hospital, Peking Union Medical College \& Chinese Academy of Medical Sciences, Beijing 100730, China. Email: lvke@163.com.

Background: To observe and assess the diagnostic value of the venous phase enhancement patterns of contrast-enhanced ultrasounds (CEUS) in patients with solid pancreatic lesions that show iso- to hyperenhancement in the arterial phase of the CEUS.

Methods: Patients who underwent CEUS for pancreatic lesions in the Peking Union Medical College Hospital between April 2017 and August 2019 were identified from the dynamic CEUS imaging database in the hospital. A total of 57 patients with pathologically or clinically diagnosed pancreatic lesions were retrospectively included in this study, and the CEUS images from these patients were evaluated. The enhancement patterns in each phase were analyzed, and each lesion was classified as malignant or benign using a five-point scale of confidence based on morphology, boundary, vascular invasion, blood flow, and enhancement patterns in ultrasound (US) and CEUS images.

Results: Rapid washout in the venous phase of CEUS images was detected in 30 malignant lesions and 4 benign lesions with malignant potential. The specificity for determining malignancy was $77.8 \%$. Continuous isoenhancement with normal pancreatic tissues in the venous phase achieved a high specificity of $95.0 \%$ for determining benign lesions. Early hyperenhancement in the arterial phase followed by slow washout in the venous phase showed high specificity of $98.1 \%$ for determining metastasis. The diagnostic confidence improved after reviewing CEUS scans (area under the receiver operating characteristic curve was 0.737 for baseline US and 0.910 for CEUS; $\mathrm{P}=0.056$ ).

Conclusions: Enhancement patterns in the venous phase of CEUS images were beneficial in the differential diagnosis of solid pancreatic lesions with iso- to hyperenhancement in the arterial phase on CEUS.

Keywords: Ultrasonography; contrast media; pancreatic neoplasms; pancreatitis; retrospective studies

Submitted Nov 09, 2020. Accepted for publication May 13, 2021.

doi: 10.21037 /qims-20-1248

View this article at: https://dx.doi.org/10.21037/qims-20-1248 


\section{Introduction}

Ultrasound (US) is widely used to screen for pancreatic lesions due to its advantages of being fast, inexpensive, and radiation-free. Its diagnostic ability has been greatly improved by introducing US contrast agents (1), such as microbubbles composed of a gas core and a stabilizing shell, that enable visualization of the blood vessels in tissues without entering the extravascular space.

In patients with solid pancreatic lesions, contrast-enhanced ultrasound (CEUS) has been widely used as it can reliably identify pancreatic ductal adenocarcinomas (PDAC) (2), which is the most lethal type of pancreatic cancer. The pooled sensitivity and specificity have been reported to be $91 \%$ and $87 \%$, respectively (3). Hypoenhancement in all phases relative to the rest of the pancreatic parenchyma is a dominant feature of PDAC on CEUS. This pattern accounts for approximately $90 \%$ of all PDAC cases $(2,4)$. However, iso- and hyperenhanced lesions remain challenging to identify, and few studies have focused on such cases.

This research was conducted to observe the characteristics of focal pancreatic lesions with iso- to hyperenhancement in the arterial phase. This investigation provides new insights into the initial differential diagnosis of focal pancreatic lesions with iso- to hyperenhancement in the arterial phase on CEUS and insights into the preliminary identification of these masses based on their enhancement patterns the venous phase.

\section{Methods}

\section{Patients}

This retrospective study was conducted following the Declaration of Helsinki (as revised in 2013). All experimental protocols were reviewed and approved by the institutional review board of the Peking Union Medical College Hospital, and the requirement for written informed consent was waived. Our institution's dynamic CEUS imaging database was reviewed, and patients who underwent CEUS for pancreatic lesions in the Peking Union Medical College Hospital between April 2017 and August 2019 were identified. A total of 598 records were retrieved. The images, videos, and medical histories were viewed by two physicians (with 11 and 6 years of experience in the abdominal US, respectively). Patients were included in this study if they presented with: (I) a solid pancreatic lesion or lesions on the US as confirmed by two physicians;
(II) lesions that were iso- to hyperenhanced in the arterial phase; and (III) a clear diagnosis for the pancreatic lesion. If the patient had several US and CEUS scans during the study, only the first scan was included. For clinical diagnosis, all lesions must be confirmed by at least 2 methods, such as contrast-enhanced computed tomography (CT), magnetic resonance imaging (MRI), or positron emission tomography-CT (PET-CT). Benign lesions must fulfill the following criteria: no marked changes in size during the follow-up periods ( $>1$ year); and no new lesions present. The following exclusion criteria were applied: (I) patients lacking a definitive diagnosis; (II) unqualified images; (III) patients with a clinical diagnosis as benign but the follow-up period was not sufficient; and (IV) patients with incomplete follow-up data. A total of 57 patients were included in this study (Figure 1), of which pathological surgical results diagnosed 28 patients, 21 were diagnosed by puncture pathological biopsy, and 8 were diagnosed by comprehensive clinical diagnosis.

\section{US and CEUS}

US and CEUS were performed by 2 trained examiners with more than 10 years experience in the US and more than 5 years experience in CEUS. The examiners were specialized in diagnostic ultrasounds for abdominal diseases. The routine CEUS protocol for the pancreas at our institution consists of US and CEUS. All scans were performed with a Philips IU-22 scanner (Philips Healthcare, Bothell, WA, USA) equipped with a $2-5 \mathrm{MHz}$ convex probe, and a mechanical index of 0.07 was used for the CEUS examination. SonoVue microbubbles (Bracco, Milan, Italy) were dissolved in saline according to the manufacturer's instructions. A fast bolus injection of $2.4 \mathrm{~mL}$ SonoVue was administered intravenously, followed by a $5 \mathrm{~mL}$ saline flush.

The patients were instructed to fast for a minimum of 6 hours prior to the procedure and were scanned in the supine position. The positions, echoes, sizes, boundaries, and blood flows of the pancreatic lesions were first recorded with a B-mode US. CEUS was then performed immediately. The maximum possible layer of the lesion was selected and CEUS was performed. The surrounding pancreatic parenchyma was included in the images as a control. The patients were instructed to maintain smooth and regular breathing patterns during the observation time, during which perfusion into the lesion was observed in real-time and the observation period was no less than 


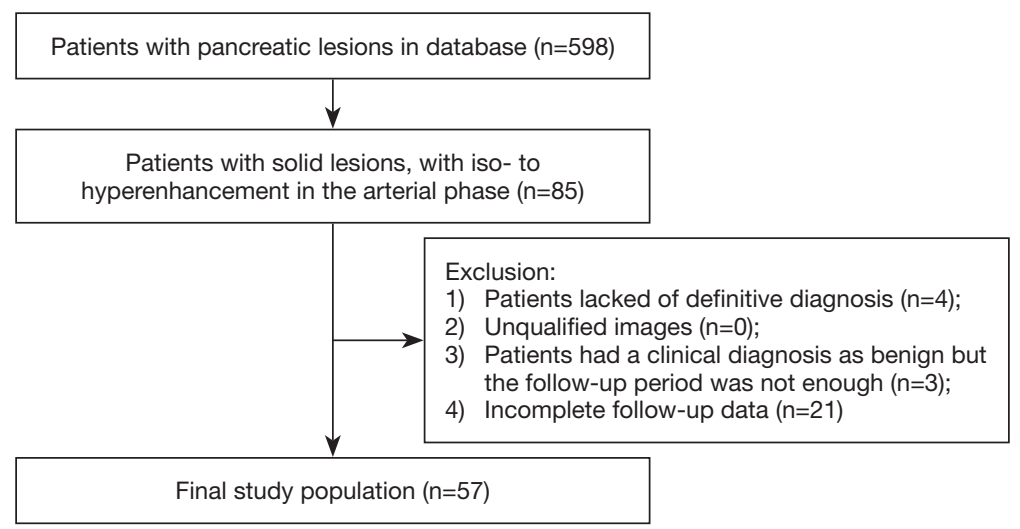

Figure 1 A flow diagram showing the patient selection criteria.

120 seconds. Dynamic images were stored for further analysis. No discomfort or adverse effects were reported after the examinations.

Image analysis was performed by an experienced physician with more than 15 years of experience in pancreatic US who was blinded to the diagnosis. Analysis was performed with the aid of time-intensity curves (TICs) drawn by the QLAB software (version 10.8, Philips Healthcare, Andover, MA). The physician was blinded to any information relating to the patient except for the US images and the CEUS videos. In each case, two regions of interest (ROIs) were selected from the lesions and normal pancreatic tissues, and two TICs were obtained. Curve fitting was performed, and the time-to-peak, peak intensity, and half-life time were estimated using the QLAB software. The ROI was square shaped with a side length of $5 \mathrm{~mm}$. The two ROIs for each case were located at a similar depth where possible. Representative areas were selected, avoiding blood vessels and interfaces. For patients with multiple but similar lesions, the enhancement patterns were mainly determined according to the largest lesion. The CEUS process was divided into the arterial phase and the venous phase, defined as $<30$ and $31-120$ seconds after injection of the contrast agent, respectively. Enhancement was classified as hyperenhancement, isoenhancement, or hypoenhancement relative to the rest of the pancreatic parenchyma. In the arterial phase, enhancement that appeared in the lesion earlier than in the pancreatic parenchyma was defined as early enhancement (Figure $2 A, B$ ). In the venous phase, the washout of the lesion faster than, slower than, or equal to that of the pancreatic parenchyma was defined as rapid washout, slow washout, and isoenhancement, respectively. Thus, the enhancement patterns in the venous phase were classified as rapid washout, slow washout, continuous isoenhancement, continuous hyperenhancement, and others (Figure $3 A, B, C, D$, Videos 1-4). Enhancement patterns were mainly defined directly with the naked eye, with the aid of TICs. Another experienced physician with more than 10 years of experience in the pancreatic US, blinded to the diagnosis, reviewed all the image analyses with no resultant objections.

Two readers (both with more than 10 years of experience in the abdominal US and more than 5 years of experience in pancreatic CEUS) characterized the lesions as benign or malignant. Before analysis, the readers were made aware of the results and conclusions of the previous part of this research. There were two sections to the scoring procedure. First, baseline US images, including the gray-scale US with color Doppler imaging, were analyzed, and the lesions were classified as benign or malignant using a 5 -point diagnostic confidence scale (Figure $4 A, B$ ) as previously described $(5-7)$. The scale is based on morphology, boundary, vascular invasion, and other image findings in the baseline US images. Second, the CEUS images of the same patient were analyzed by the readers. The score was then reassessed based on the results and conclusions of the previous part of this research and the enhancement patterns in the CEUS. A consensus is then reached by discussion.

\section{Statistical analysis}

Categorical and ordinal variables were expressed as counts and proportions, and quantitative variables were expressed as mean \pm standard deviation if the data were normally distributed. Statistical analyses were performed using SPSS statistical software version 25.0 (SPSS Inc., Chicago, IL, 

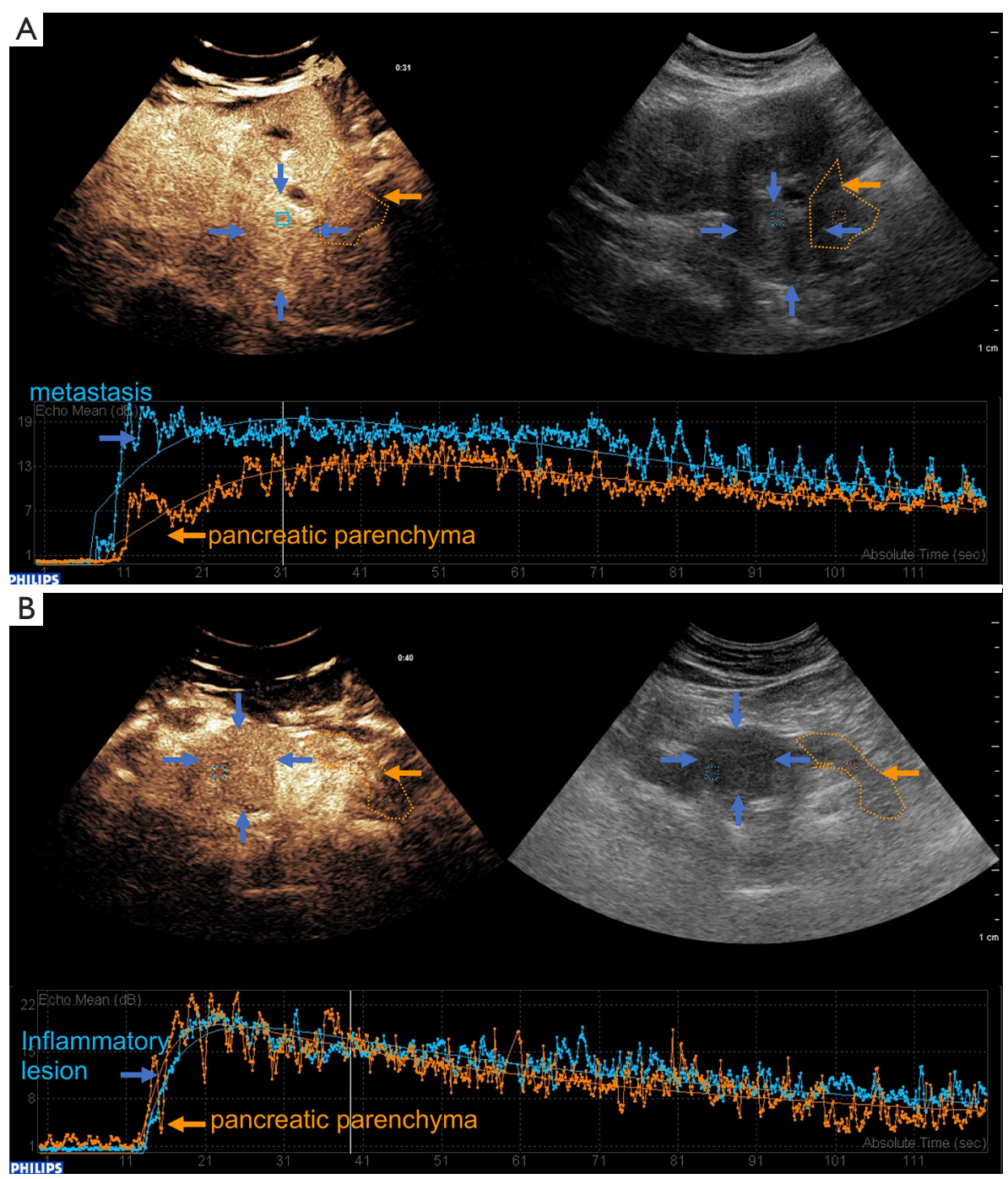

Figure 2 Contrast-enhanced ultrasound images of the early and not-early enhancement in the arterial phase. (A) A lesion classified with early hyperenhancement in the arterial phase, diagnosed as pancreatic metastasis of clear cell renal cell carcinoma in a 53-year-old woman. In the arterial phase, enhancement in the lesion (blue arrows in the CEUS image and blue curve in the TICs) clearly occurred earlier than that in the pancreatic parenchyma (orange arrows and orange curve) (time-to-peak estimated to be 32.9 to 43.2 s). The enhancement intensity in the lesion was much higher than that in the pancreatic parenchyma (peak intensity estimated to be 19.2 to $13.1 \mathrm{~dB}$ ). (B) A lesion classified with isoenhancement with not-early enhancement in the arterial phase, diagnosed as an inflammatory lesion in a 62 -year-old man. In the arterial phase, the enhancement of the lesion (blue arrows and blue curve) did not occur earlier than that in the pancreatic parenchyma (orange arrows and orange curve) (time-to-peak estimated to be 26.6 to $22.7 \mathrm{~s}$ ). The enhancement intensity was similar between the lesion and pancreatic parenchyma (peak intensity estimated to be 17.9 to $18.5 \mathrm{~dB}$ ). CEUS, contrast-enhanced ultrasound; TIC, time intensity curve.

USA). The $\chi^{2}$ test was used to assess the differences of categorical variables. The difference of normally distributed continuous variables between two groups was analyzed using the Student's $t$-test, and the Mann-Whitney U test was used for comparing abnormally distributed continuous variables. Logistic regression was used for intergroup comparisons of location. The diagnostic performances of baseline US and CUES were calculated using a non-parametric receiver operating characteristic (ROC) curve and compared using the $Z$ test of the area under curve (AUC). The accuracy (ACC), sensitivity (SEN), specificity (SPE), positive predictive value (PPV), negative predictive value (NPV), 

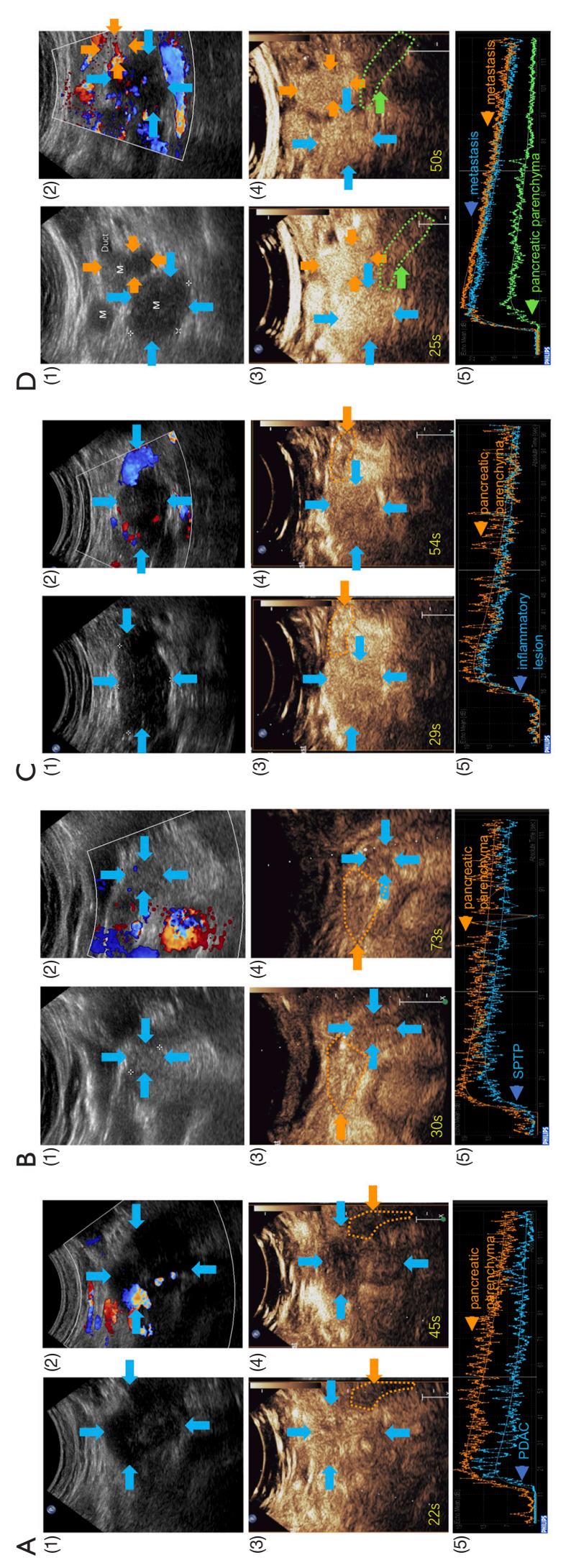

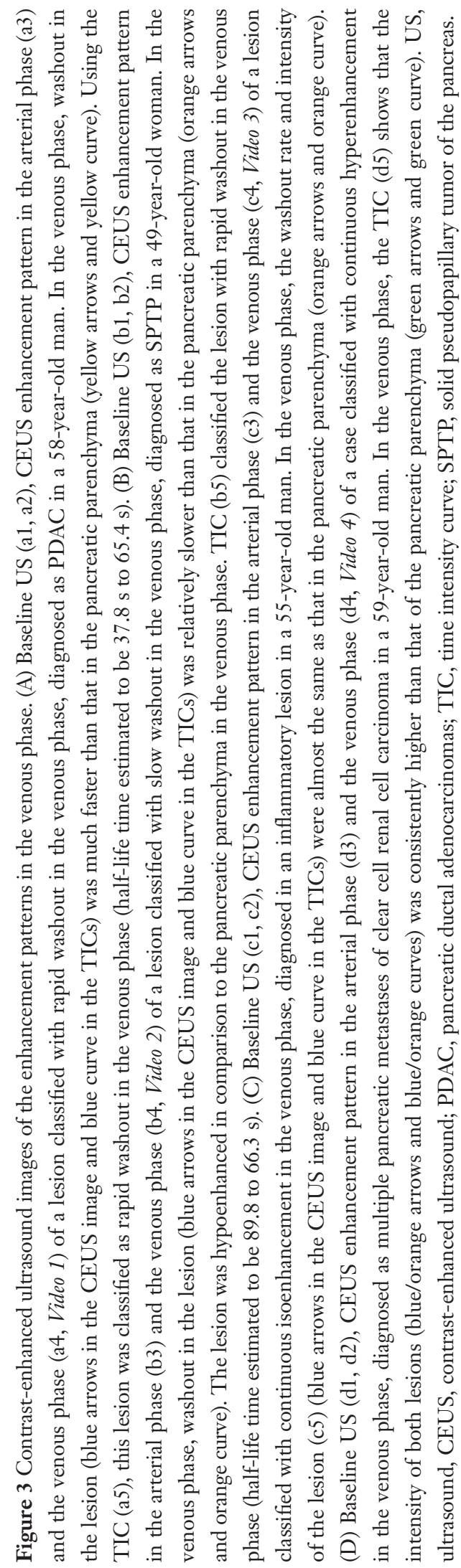




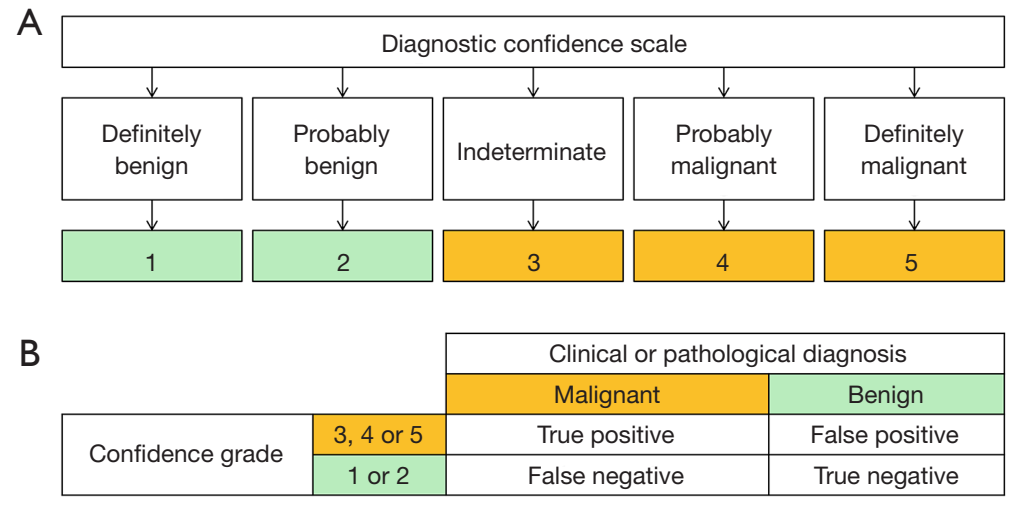

Figure 4 Schematic diagram of the 5-point diagnostic confidence scale. (A) A 5-point diagnostic confidence scale was assigned for each case as follows: 1 , definitely benign; 2, probably benign; 3, indeterminate; 4, probably malignant; and 5, definitely malignant. (B) The retrospectively determined benign or malignant diagnoses were considered: true-positive if the lesion was correctly assessed as malignant, with an assigned confidence grade of 3,4 or 5; false-negative if the lesion was assigned a confidence grade of 1 or 2 and incorrectly assessed as benign; true-negative if the lesion was assigned a confidence grade of 1 or 2 and correctly assessed as benign; or false-positive if the lesion was assigned a confidence grade of 3,4 , or 5 and incorrectly assessed as malignant.

Table 1 The baseline characteristics of patients and lesions between the malignant and benign groups

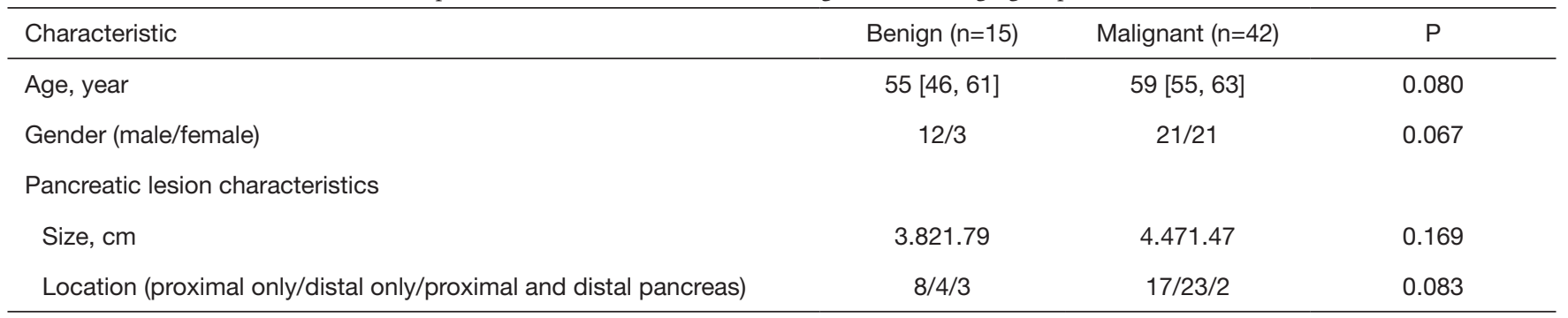

positive likelihood ratio $(\mathrm{LR}+)$, and negative likelihood ratio ( $\mathrm{LR}_{-}$) were calculated using the $2 \times 2$ contingency table. A two-sided $\mathrm{P}$ value $<0.05$ was considered statistically significant.

\section{Results}

\section{Patient characteristics}

A total of 57 patients (33 males and 24 females) were recruited for this study. Table 1 provides the patient demographics and baseline characteristics. Age, gender, lesion size, and lesion location did not differ significantly between patients with benign lesions and malignant lesions.

\section{Clinical and pathological diagnosis}

Table 2 summarizes the clinical and pathological diagnoses and enhancement patterns. PDAC accounted for most of the diagnoses. Other pathological findings included metastasis, pancreatic neuroendocrine neoplasm (p-NEN), inflammation, intraductal papillary mucinous neoplasm (IPMN), solid pseudopapillary tumor of the pancreas (SPTP), and so on. Four metastases originated from renal clear cell carcinoma, and one originated from small cell lung cancer (SCLC).

\section{Characteristics of the CEUS enhancement patterns}

The characteristics of the CEUS enhancement patterns of the lesions are summarized in Table 3. The distribution of hyperenhancement and isoenhancement in the arterial phase was not significantly different between patients with benign lesions and patients with malignant lesions. In some of the lesions, the contrast agent washed in rapidly, and the CEUS 
Table 2 The final clinical/pathological diagnosis and enhancement patterns of malignant and benign lesions

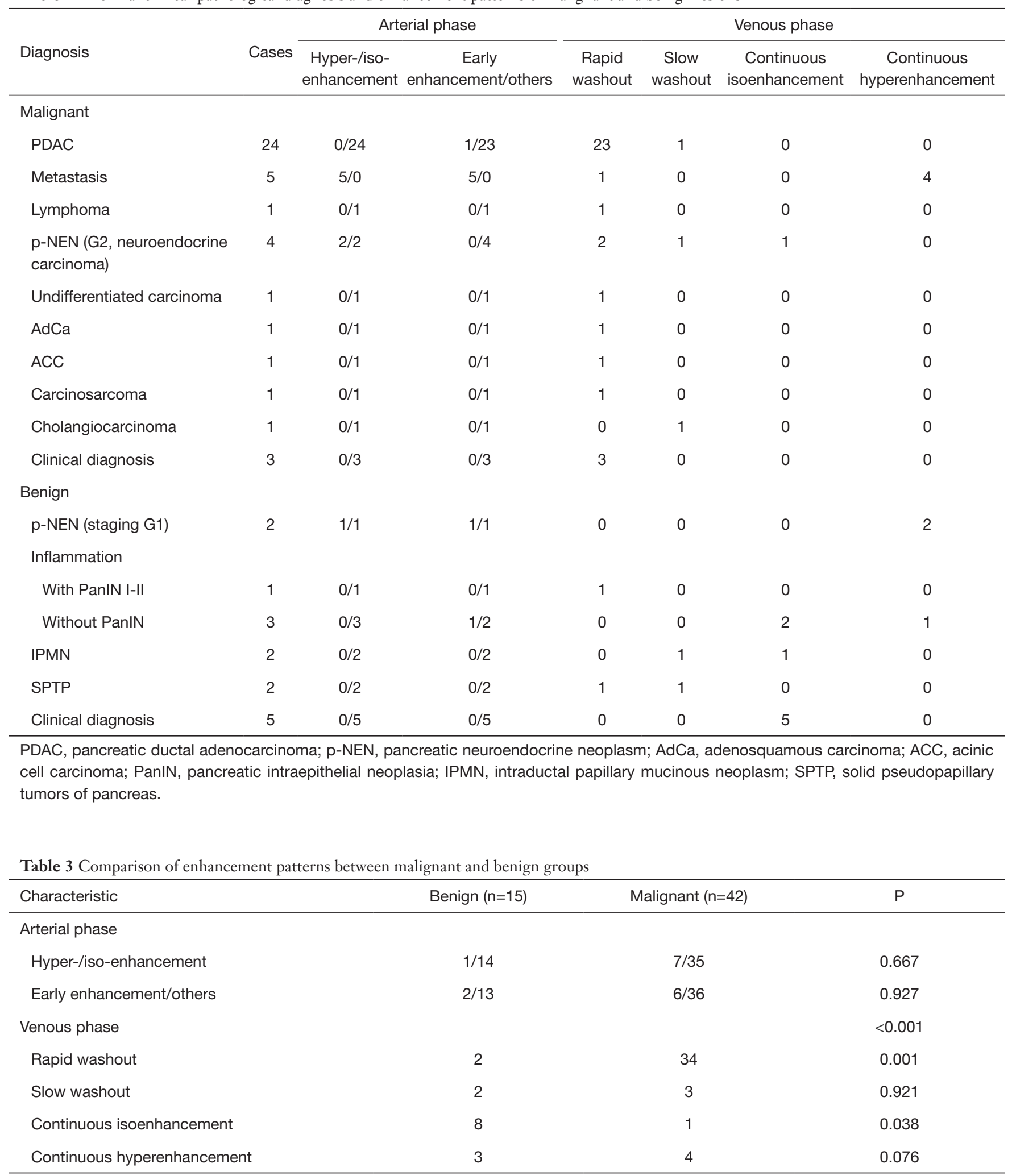


images presented early enhancement in the arterial phase. The distribution of this characteristic showed no significant difference between benign and malignant patients.

The CEUS characteristics in the venous phase provided more information for differential diagnosis. According to the enhancement patterns in the venous phase, the lesions were divided into 4 categories. Among these, the distributions of rapid washout $(\mathrm{P}=0.001)$ and continuous isoenhancement $(\mathrm{P}=0.038)$ were significantly different between the benign and malignant groups.

\section{The diagnostic value of rapid washout in the venous phase for malignancy}

Rapid washout in the venous phase was detected in 34 malignant lesions and 2 benign lesions. This pattern was distributed differently between the two groups $(\mathrm{P}<0.001)$, with a sensitivity of $81.0 \%$ and a specificity of $86.7 \%$ for determining malignancy. The diagnoses of the 34 malignant lesions with rapid washout in the venous phase were PDAC (23 cases), clinical diagnosis ( 3 cases), p-NEN ( 2 cases), carcinosarcoma, lymphoma, undifferentiated carcinoma, adenosquamous carcinoma (AdCa), acinic cell carcinoma (ACC), and metastasis (1 case each).

The pathological diagnoses of the 2 benign lesions with rapid washout in the venous phase were SPTP and inflammation with focal pancreatic intraepithelial neoplasia (PanIN) I-II, both of which have malignant potential.

\section{The diagnostic value of continuous isoenhancement in the venous phase for benign lesions}

Continuous isoenhancement with the presence of normal pancreatic tissues in the venous phase was observed in 8 benign lesions and 1 malignant lesion (determined as neuroendocrine carcinoma based on a pathological diagnosis). This pattern was distributed differently between these two groups, with a high specificity of $97.6 \%$ and a relatively low sensitivity of $53.3 \%$ for determining benign lesions.

\section{The diagnostic value of continuous hyperenhancement in the venous phase for metastasis}

Continuous hyperenhancement in the venous phase was found in 3 benign lesions and 4 malignant lesions. The distribution of this pattern was not different between the two groups, but specificity for metastasis was noted. The
7 lesions with this feature consisted of 4 metastases, 2 p-NENs, and 1 inflammation. Among all 5 metastases, only 1 case originating from SCLC showed rapid washout rather than continuous hyperenhancement in the venous phase. Continuous hyperenhancement in the venous phase had a relatively low sensitivity of $80.0 \%$ and a high specificity of 94.2\% for determining metastasis $(\mathrm{P}<0.001)$.

\section{The diagnostic value of early byperenhancement in the arterial phase followed by continuous hyperenhancement in the venous phase for metastasis}

Early hyperenhancement in the arterial phase alone showed no difference in distribution between the benign and malignant groups, but this pattern could help further improve the specificity of determining metastasis. As mentioned before, 7 cases were found with continuous hyperenhancement in the venous phase, including 4 metastases, 2 p-NENs, and 1 case of inflammation. In these cases, the 4 metastases originated from renal clear cell carcinoma. One p-NEN presented early hyperenhancement in the arterial phase. The other p-NEN and one inflammatory lesion presented with isoenhancement. Thus, early hyperenhancement in the arterial phase followed by continuous hyperenhancement in the venous phase achieved a high specificity of $98.1 \%$ for determining metastasis, but the sensitivity remained relatively low at $80.0 \%(\mathrm{P}<0.001)$.

\section{A comparison of confidence grade after reviewing baseline US and CEUS images}

After reviewing the baseline US images and CEUS scans, lesions were classified by the readers as benign (confidence grade 1 or 2 ) or malignant (confidence grade 3,4 , or 5 ) according to their diagnostic confidence, with the above results taken into consideration. After reviewing the CEUS scans, the confidence grade (Table 4) changed with statistical significance $(\mathrm{P}=0.030)$. More lesions had definitive and correct diagnoses. In benign lesions with an assigned confidence grade of 1 , the correct diagnosis was achieved in $0 \%$ of cases after baseline US, and this increased to $53.3 \%$ after CEUS review. In malignant lesions with an assigned confidence grade of 5 , the correct diagnosis was achieved in $14.3 \%$ of cases after baseline US, and this increased to $88.1 \%$ after CEUS review. The ACC, SEN, SPE, PPV, NPV, LR+, and LR- for diagnosing malignancy all improved following a review of the CEUS scans (Table 5). The area under the ROC curve (Figure 5, Table 5) increased 
Table 4 Comparison of confidence grade after reviewing baseline US and CEUS

\begin{tabular}{lccc}
\hline & Benign (\%) & Malignant (\%) & $\mathrm{P}$ \\
\hline Baseline US only & & & $<0.0001$ \\
1 & $0(0.0)$ & $0(0.0)$ & \\
2 & $3(20.0)$ & $4(9.5)$ & \\
3 & $8(53.3)$ & $8(19.0)$ & \\
4 & $4(26.7)$ & $24(57.1)$ & \\
5 & $0(0.0)$ & $6(14.3)$ & \\
CEUS & & & \\
1 & $8(53.3)$ & $1(2.4)$ & \\
2 & $4(26.7)$ & $0(0.0)$ & \\
3 & $1(6.7)$ & $1(2.4)$ & \\
4 & $0(0.0)$ & $3(7.1)$ & \\
5 & $2(13.3)$ & $37(88.1)$ & \\
\hline
\end{tabular}

US, ultrasound; CEUS, contrast-enhanced ultrasound.

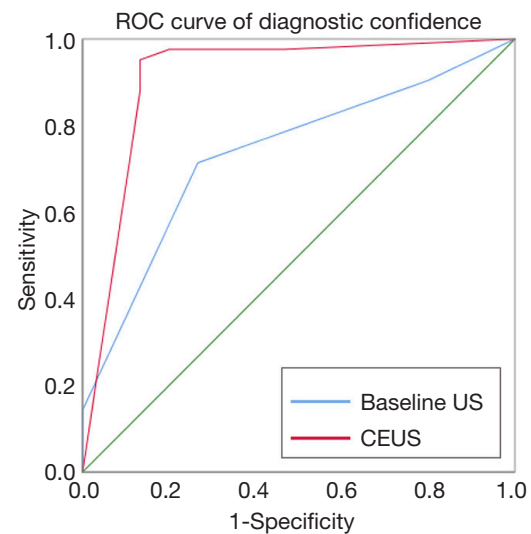

Figure 5 A graphical representation of the increase in diagnostic confidence after a review of contrast-enhanced ultrasound scans. The ROC curve is plotted to discriminate between benign and malignant lesions after a review of the baseline US scans (blue line) and after a review of the CEUS scans (red line). ROC, receiver operating characteristic; US, ultrasound, CEUS, contrastenhanced ultrasound.

Table 5 Diagnostic value of baseline US and CEUS in solid pancreatic lesions with iso- to hyperenhancement in the arterial phase on CEUS

\begin{tabular}{lcccccccc}
\hline & ACC (\%) & SEN (\%) & SPE (\%) & PPV (\%) & NPV (\%) & PLR & NLR & AUC \\
\hline Baseline US only & 71.93 & 90.48 & 20.00 & 76.00 & 42.86 & 1.13 & 0.48 & 0.737 \\
CEUS & 92.98 & 97.62 & 80.00 & 93.18 & 92.31 & 4.88 & 0.03 & 0.910 \\
\hline
\end{tabular}

US, ultrasound; CEUS, contrast-enhanced ultrasound; ACC, accuracy; SEN, sensitivity; SPE, specificity; PPV, positive predictive value; NPV, negative predictive value; PLR, positive likelihood ratio; NLR, negative likelihood ratio; AUC, area under the ROC curve.

from 0.737 for baseline US to 0.910 for CEUS; however, the difference was not statistically significant $(\mathrm{z}=1.908$, $\mathrm{P}=0.056$ ).

\section{Discussion}

This retrospective study provided an overview of solid pancreatic lesions with iso- to hyperenhancement in the arterial phase on CEUS. There were several vital findings (Figure 6). First, rapid washout in the venous phase is a reliable indicator of malignancy and malignant potential and has high specificity. Second, isoenhancement in the venous phase is highly specific for determining benign lesions. Third, early hyperenhancement in the arterial phase followed by hyperenhancement in the venous phase has a high specificity for determining metastasis. With these findings, the diagnostic performance of pancreatic lesions can be enhanced, thereby improving the diagnostic confidence.

PDAC has been reported to be hypovascular in 73-93\% of cases, and thus, most PDAC cases can be identified by typical hypoenhancement in all phases with reliable accuracy $(1,3,8)$. However, it should be noted that there are a fraction of PDAC cases that show iso- and hyperenhancement in the arterial phase, and it is challenging to distinguish iso- or hyperenhanced cases of PDAC from other lesions. Therefore, it is necessary to make comprehensive judgments and diagnoses while considering the enhancement pattern in the venous phase. However, there is a paucity of information in the literature on this topic.

This study indicated that rapid washout in the venous phase can be a predictor of malignancy in lesions with iso- to hyperenhancement in the arterial phase. Few studies have focused on the use of the rapid washout to differentiate between malignant and benign lesions. In addition, it should be noted that the pathological 


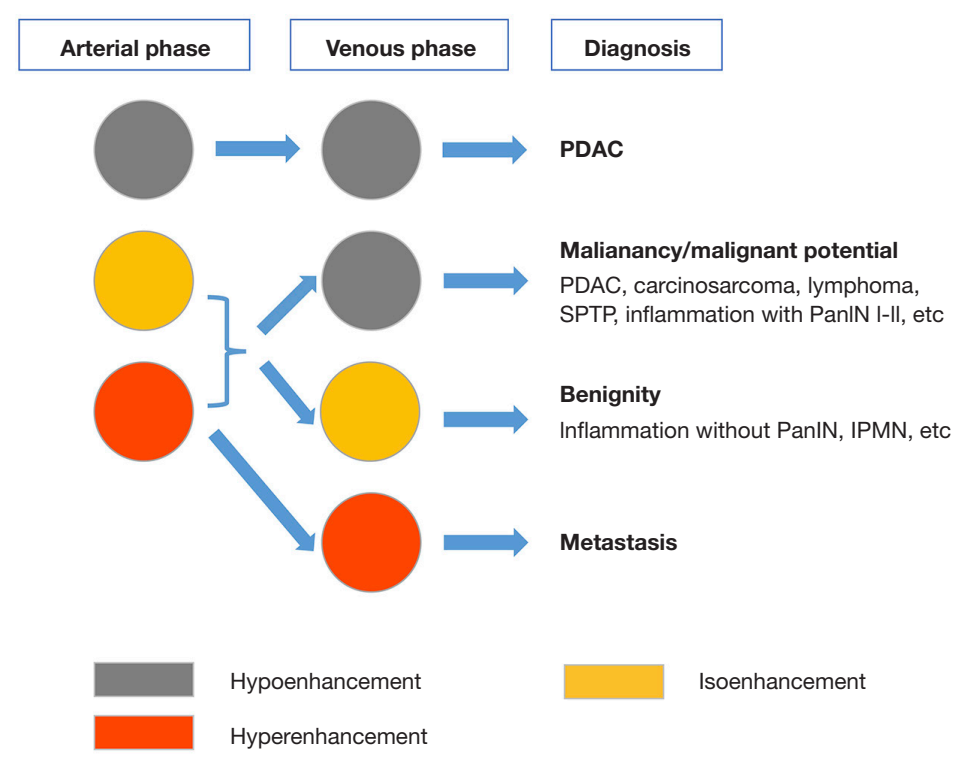

Figure 6 Schematic representation of the findings in this report. Previous studies have suggested that hypoenhancement in all phases relative to the rest of the pancreatic parenchyma is a dominant feature of PDAC in CEUS. This study determined that for solid pancreatic lesions with iso- to hyperenhancement in the arterial phase, rapid washout in the venous phase is a reliable indicator of malignancy and malignant potential with a high specificity, and isoenhancement in the venous phase is highly specific for determining benign lesions. In addition, early hyperenhancement in the arterial phase followed by hyperenhancement in the venous phase has a high specificity for determining metastasis. PDAC, pancreatic ductal adenocarcinomas; CEUS, contrast-enhanced ultrasound; IPMN, intraductal papillary mucinous neoplasm; PanIN, pancreatic intraepithelial neoplasia.

diagnoses of the 4 benign lesions with rapid washout in the venous phase were p-NEN G2, SPTP, and inflammation with focal PanIN I-II, all of which have malignant potential. The rapid wash out possible basis might be arteriovenous shunts and the disorganized vessel networks in malignant lesions (1). Unlike the contrast media applied for CT or MRI, the microbubble contrast agents used in CEUS are intravascular contrast agents without an extravascular phase. Thus, the late phase of CEUS does not correspond to the interstitial or parenchymal equilibrium phase described on CT or MRI (8). Hence, a malignant diagnosis must be considered when rapid washout in the venous phase is observed, regardless of the enhancement pattern in the arterial phase, and further attempts at differential diagnosis and pathological diagnosis should be made.

The present results will be of significance for the percutaneous biopsy of pancreatic lesions. Many studies have shown that percutaneous ultrasound-guided biopsies have a relatively low NPV (57.0-76.3\%) (9-11). This means that false-negative cases, in which a lesion harbors malignant cells but shows negative results on biopsy, require more attention. To avoid missed diagnoses, a lesion with rapid washout in the venous phase on CEUS may need further examination, close clinical follow-up, and even repeated biopsy, despite an initial negative biopsy result. On the other hand, CEUS that shows the liquid or necrotic areas inside the pancreatic mass prior to USguided biopsy may help identify the target. Sampling yield has been reported to be improved (accuracy of $78 \% v s$. $86 \%$ ) when CEUS was applied to guide pancreatic fineneedle aspiration (FNA) (12), and the diagnostic accuracy was also improved in other organs, such as the liver, lungs, and prostate (13-15). Despite the presence of liquid and necrotic areas, it was shown that nearly $50 \%$ of PDAC cases were accompanied by surrounding chronic pancreatitis (16). Sometimes it is challenging to differentiate pancreatitis from PDAC. Although CEUS can improve the differential diagnosis by showing hypoenhancement in PDAC and iso- to hyperenhancement in inflammatory lesions (17), the intensity of the enhancement tended to be lower along with the chronic and longstanding inflammatory process, probably due to the progression of fibrosis (18-20). Relying on the enhancement patterns in the arterial phase alone 
may not lead to a clear distinction. According to our results, rapid washout in the venous phase appeared in only $25 \%$ of cases of inflammation and favored (potentially) malignant lesions. Considering its specificity for malignancies in this study, rapid washout in the venous phase might be an essential sign for guiding biopsy. Choosing an area with this sign as a biopsy target might help avoid the interference from inflammation, improve the representation of the samples, and increase the accuracy of target identification.

Endoscopic ultrasound (EUS) and EUS-guided fineneedle aspiration (EUS-FNA) are recommended in many guidelines for solid pancreatic lesions (21). EUS is regarded as one of the most sensitive imaging modalities for the detection of pancreatic lesions, and contrastenhanced-EUS (CE-EUS) can improve the specificity of pancreatic cancer diagnosis $(53 \%$ for EUS vs. $88 \%$ for CE-EUS) (22). In recent research, EUS-FNA with contrast-enhanced harmonic imaging increased the rates of adequate sampling and sensitivity compared to EUSFNA with fundamental B mode imaging $(84.9 \%$ vs. $68.8 \%$, $\mathrm{P}=0.003$ and $76.5 \%$ vs. $58.8 \%, \mathrm{P}=0.011$, respectively) (23). However, additional studies are required to support this conclusion. The diagnostic accuracy of percutaneous USFNA is comparable to EUS-FNA (24), and the incidence of complications associated with EUS or US guidance range from $0 \%$ to $5 \%$ (25). In our hospital, the percutaneous ultrasound-guided puncture was more often selected due to the advantages of the short duration of the appointments and low costs. This current research demonstrated that the enhancement pattern of the venous phase in CEUS might have a promising role in differential diagnosis and biopsy target selection. Furthermore, the enhancement pattern of the venous phase may have implications for differential diagnosis and biopsy target selection in CE-EUS and EUSFNA.

The majority of p-NENs are hypervascular, which is the essential feature of p-NENs on CEUS (26). Studies have reported that the CEUS pattern correlates with tumor grading in p-NENs and might be a prognostic and predictive factor for the responsiveness to somatostatin analog therapy (27). In our study, continuous isoenhancement in the venous phase-only was presented in a neuroendocrine carcinoma case. None of the other 4 p-NEN cases (stages G1 and G2) showed a similar pattern. This suggested that the relationship of enhancement patterns in the venous phase with staging and the prognostic assessment of p-NENs may be worth exploring.

Metastasis and many other lesions with abundant arterialization, such as pNETs, present as hyperenhancing lesions in the arterial phase on CEUS examinations $(2,28)$, leading to difficulties in the differential diagnosis. In this study, early hyperenhancement in the arterial phase followed by continuous hyperenhancement in the venous phase had a high specificity $(98.1 \%)$ for determining metastasis. Additionally, enhancement patterns in the venous phase may be a significant factor in the differential diagnosis of these hypervascular lesions.

Although currently, CTs and MRIs are considered more effective than CEUS, Ran and colleagues noted that the average cost of CTs and MRIs is at least double that of CEUS in China (29). Additionally, the limitations of regional economic development or medical and health facilities should be taken into consideration. Some patients cannot receive contrast-enhanced CT scans or contrastenhanced MRI examinations due to contraindications (such as kidney failure, allergies to iodine for CT contrast agents, claustrophobia, and pacemakers and metal objects in the body that are unsuitable for MRI). Thus, CEUS may provide a novel option for the differential diagnosis of solid pancreatic lesions.

There were some limitations to this study. First, this was a single-center retrospective study from a third-grade Class A hospital in Beijing, China, with potential selection bias and limited generalizability. Second, the sample size was relatively small. Further prospective studies with larger cohorts are warranted to confirm these findings.

In conclusion, this investigation demonstrated that enhancement patterns in the venous phase improved the diagnostic performance for solid pancreatic lesions with isoto hyperenhancement in the arterial phase on CEUS.

\section{Acknowledgments}

Funding: This study was funded by the National Natural Science Foundation of China (No. 81873902); the Chinese Academy of Medical Sciences Innovation Fund for Medical Sciences (No. 2016-I2M-3-005); and the Chinese Academy of Medical Sciences Innovation Fund for Medical Sciences (CIFMS) (No. 2020-I2M-C\&T-B-039).

\section{Footnote}

Conflicts of Interest: All authors have completed the ICMJE uniform disclosure form (available at https://dx.doi. org/10.21037/qims-20-1248). The authors have no conflicts of interest to declare. 
Ethical Statement: The authors are accountable for all aspects of the work in ensuring that questions related to the accuracy or integrity of any part of the work are appropriately investigated and resolved. The study was conducted in accordance with the Declaration of Helsinki (as revised in 2013). The study was approved by the institutional review board of the Peking Union Medical College Hospital (No.: SK-1139) and individual consent for this retrospective analysis was waived.

Open Access Statement: This is an Open Access article distributed in accordance with the Creative Commons Attribution-NonCommercial-NoDerivs 4.0 International License (CC BY-NC-ND 4.0), which permits the noncommercial replication and distribution of the article with the strict proviso that no changes or edits are made and the original work is properly cited (including links to both the formal publication through the relevant DOI and the license). See: https://creativecommons.org/licenses/by-nc-nd/4.0/.

\section{References}

1. Fan Z, Li Y, Yan K, Wu W, Yin S, Yang W, Xing B, Li X, Zhang X. Application of contrast-enhanced ultrasound in the diagnosis of solid pancreatic lesions--a comparison of conventional ultrasound and contrast-enhanced CT. Eur J Radiol 2013;82:1385-90.

2. Sidhu PS, Cantisani V, Dietrich CF, Gilja OH, Saftoiu A, Bartels E, et al. The EFSUMB Guidelines and Recommendations for the Clinical Practice of ContrastEnhanced Ultrasound (CEUS) in Non-Hepatic Applications: Update 2017 (Long Version). Ultraschall Med 2018;39:e2-e44.

3. Li XZ, Song J, Sun ZX, Yang YY, Wang H. Diagnostic performance of contrast-enhanced ultrasound for pancreatic neoplasms: A systematic review and metaanalysis. Dig Liver Dis 2018;50:132-8.

4. Claudon M, Cosgrove D, Albrecht T, Bolondi L, Bosio $\mathrm{M}$, Calliada F, et al. Guidelines and good clinical practice recommendations for contrast enhanced ultrasound (CEUS) - update 2008. Ultraschall Med 2008;29:28-44.

5. Sofuni A, Tsuchiya T, Itoi T. Ultrasound diagnosis of pancreatic solid tumors. J Med Ultrason (2001) 2020;47:359-76.

6. Dietrich CF, Sahai AV, D'Onofrio M, Will U, Arcidiacono PG, Petrone MC, Hocke M, Braden B, Burmester E, Möller K, Săftoiu A, Ignee A, Cui XW, Iordache S, Potthoff A, Iglesias-Garcia J, Fusaroli P, Dong Y, Jenssen
C. Differential diagnosis of small solid pancreatic lesions. Gastrointest Endosc 2016;84:933-40.

7. D'Onofrio M, Barbi E, Dietrich CF, Kitano M, Numata K, Sofuni A, Principe F, Gallotti A, Zamboni GA, Mucelli RP. Pancreatic multicenter ultrasound study (PAMUS). Eur J Radiol 2012;81:630-8.

8. Scialpi M, Reginelli A, D'Andrea A, Gravante S, Falcone G, Baccari P, Manganaro L, Palumbo B, Cappabianca S. Pancreatic tumors imaging: An update. Int J Surg 2016;28 Suppl 1:S142-55.

9. Kahriman G, Ozcan N, Dogan S, Ozmen S, Deniz K. Percutaneous ultrasound-guided core needle biopsy of solid pancreatic masses: Results in 250 patients. J Clin Ultrasound 2016;44:470-3.

10. Huang Y, Shi J, Chen YY, Li K. Ultrasound-Guided Percutaneous Core Needle Biopsy for the Diagnosis of Pancreatic Disease. Ultrasound Med Biol 2018;44:1145-54.

11. Yang RY, Ng D, Jaskolka JD, Rogalla P, Sreeharsha B. Evaluation of percutaneous ultrasound-guided biopsies of solid mass lesions of the pancreas: a center's 10-year experience. Clin Imaging 2015;39:62-5.

12. Seicean A, Badea R, Moldovan-Pop A, Vultur S, Botan EC, Zaharie T, Săftoiu A, Mocan T, Iancu C, Graur F, Sparchez Z, Seicean R. Harmonic Contrast-Enhanced Endoscopic Ultrasonography for the Guidance of FineNeedle Aspiration in Solid Pancreatic Masses. Ultraschall Med 2017;38:174-82.

13. Nolsøe CP, Nolsøe AB, Klubien J, Pommergaard HC, Rosenberg J, Meloni MF, Lorentzen T. Use of Ultrasound Contrast Agents in Relation to Percutaneous Interventional Procedures: A Systematic Review and Pictorial Essay. J Ultrasound Med 2018;37:1305-24.

14. Wu W, Chen MH, Yin SS, Yan K, Fan ZH, Yang W, Dai Y, Huo L, Li JY. The role of contrast-enhanced sonography of focal liver lesions before percutaneous biopsy. AJR Am J Roentgenol 2006;187:752-61.

15. Spârchez Z, Radu P, Zaharia T, Kacso G, Grigorescu I, Badea $R$. Contrast enhanced ultrasound guidance: a new tool to improve accuracy in percutaneous biopsies. Med Ultrason 2010;12:133-8.

16. Wei Y, Yu XL, Liang P, Cheng ZG, Han ZY, Liu FY, Yu $\mathrm{J}$. Guiding and controlling percutaneous pancreas biopsies with contrast-enhanced ultrasound: target lesions are not localized on B-mode ultrasound. Ultrasound Med Biol 2015;41:1561-9.

17. D'Onofrio M, Zamboni G, Tognolini A, Malago R, Faccioli N, Frulloni L, Pozzi Mucelli R. Mass-forming pancreatitis: value of contrast-enhanced ultrasonography. 
World J Gastroenterol 2006;12:4181-4.

18. Rickes S, Unkrodt K, Neye H, Ocran KW, Wermke W. Differentiation of pancreatic tumours by conventional ultrasound, unenhanced and echo-enhanced power Doppler sonography. Scand J Gastroenterol 2002;37:1313-20.

19. Rickes S, Unkrodt K, Ocran K, Neye H, Wermke W. Differentiation of neuroendocrine tumors from other pancreatic lesions by echo-enhanced power Doppler sonography and somatostatin receptor scintigraphy. Pancreas 2003;26:76-81.

20. Fan Z, Yan K, Wu W, Yin S, Dai Y, Yang W, Chen M. Quantitative analysis with CEUS in differential diagnosis of pancreatic carcinoma and mass forming pancreatitis. Chinese J Med Imaging Technol 2012;28:1354-8.

21. Saftoiu A, Napoleon B, Arcidiacono PG, Braden B, Burmeister S, Carrara S, et al. Do we need contrast agents for EUS? Endosc Ultrasound 2020;9:361-8.

22. Kitano M, Yoshida T, Itonaga M, Tamura T, Hatamaru K, Yamashita Y. Impact of endoscopic ultrasonography on diagnosis of pancreatic cancer. J Gastroenterol 2019;54:19-32.

23. Itonaga M, Kitano M, Kojima F, Hatamaru K, Yamashita Y, Tamura T, Nuta J, Kawaji Y, Shimokawa T, Tanioka K, Murata SI. The usefulness of EUS-FNA with contrast-enhanced harmonic imaging of solid pancreatic lesions: A prospective study. J Gastroenterol Hepatol 2020;35:2273-80.

24. Horwhat JD, Paulson EK, McGrath K, Branch MS, Baillie J, Tyler D, Pappas T, Enns R, Robuck G, Stiffler H, Jowell
P. A randomized comparison of EUS-guided FNA versus CT or US-guided FNA for the evaluation of pancreatic mass lesions. Gastrointest Endosc 2006;63:966-75.

25. D'Onofrio M, De Robertis R, Barbi E, Martone E, Manfrin E, Gobbo S, Puntel G, Bonetti F, Pozzi Mucelli R. Ultrasound-guided percutaneous fine-needle aspiration of solid pancreatic neoplasms: 10-year experience with more than 2,000 cases and a review of the literature. Eur Radiol 2016;26:1801-7.

26. Lee L, Ito T, Jensen RT. Imaging of pancreatic neuroendocrine tumors: recent advances, current status, and controversies. Expert Rev Anticancer Ther 2018;18:837-60.

27. Del Prete M, Di Sarno A, Modica R, Lassandro F, Giorgio A, Bianco A, Muto M, Gasperi M, Del Prete F, Colao A, Montesarchio V, Faggiano A; ENETS Centre of Excellence Multidisciplinary Group for Neuroendocrine Tumors in Naples (Italy). Role of contrast-enhanced ultrasound to define prognosis and predict response to biotherapy in pancreatic neuroendocrine tumors. J Endocrinol Invest 2017;40:1373-80.

28. Fan Z, Yan K, Wang Y, Yin S, Yang W, Chen M. Investigation of the manifestation of pancreatic metastases on contrast-enhanced ultrasound. Chinese J Ultrason 2014;(11):948-51.

29. Ran L, Zhao W, Zhao Y, Bu H. Value of contrastenhanced ultrasound in differential diagnosis of solid lesions of pancreas (SLP): A systematic review and a metaanalysis. Medicine (Baltimore) 2017;96:e7463.
Cite this article as: Chen X, Hao F, Gui Y, Zhang J, Tan L, Xiao M, Zhang Q, Meng H, Li J, Jiang Y, Lv K. Enhancement patterns in the venous phase of contrast-enhanced ultrasounds: diagnostic value for patients with solid pancreatic lesions. Quant Imaging Med Surg 2021;11(10):4321-4333. doi: 10.21037/ qims-20-1248 